\title{
Gatekeeper incentives and demand inducement: An empirical analysis of care managers in the Japanese Long-Term Care Insurance program
}

\author{
Shinya Sugawara*and Jiro Nakamura ${ }^{\dagger}$
}

\begin{abstract}
This study analyzes the incentives and supplier-induced demand of care managers, who are intermediaries between consumers and service providers in the Japanese social insurance program for long-term care. Care managers can be considered as pure gatekeepers in that their function is limited to referral people to specialists and they themselves do not provide care. Care managers are rewarded by capitation, which is considered as a cost-effective payment mechanism for insurers. However, many care managers actually work for firms that also operate as service providers. Service providers are rewarded by a fee-for-service payment and can have a motivation to induce excess consumer demand. The violation of the neutrality of care managers might result in a financial burden on social insurance. In this study, we empirically test whether there is a positive correlation between care manager density and care costs, which might imply the existence of supplier-induced demand. Our results show a positive correlation, particularly in the case of care managers who work for firms that jointly operate in service provision sectors. Based on these results, we conduct a quantitative analysis, and show that the demand induced by care managers might produce a considerable financial burden on social insurance.
\end{abstract}

Keyword: Elderly care; Gatekeepers; Incentive; Supplier-induced demand; Japanese Long-Term Care Insurance program; Care managers

${ }^{*}$ Corresponding author. University of Tokyo, 7-3-1 Hongo, Bunkyo, Tokyo 113-0033, Japan, and Nihon University, 12-5, Gobancho, Chiyoda, Tokyo 102-8251, Japan: Email: sugawara_shinya@ci.i.utokyo.ac.jp, Tel: +81-3-5841-6899

${ }^{\dagger}$ Nihon University, 12-5, Gobancho, Chiyoda, Tokyo 102-8251, Japan: Email: nakamura.jiro@nihonu.ac.jp 
JEL classification codes: I18; I11; J13

\section{Introduction}

In response to the overwhelming concern about the country's aging population, the Japanese government established the radical Long-Term Care Insurance (LTCI) program in 2000. This mandatory program with universal coverage instantaneously created new markets for various formal elderly care services, such as home care, day care services, and short-stay care. To guide consumers in this new and complicated market, the LTCI introduced intermediaries, called care managers, between consumers and the service providers. This study investigates whether care managers promote efficiency within the social insurance program.

Care managers can be considered as gatekeepers who refer consumers to formal care providers. In the healthcare sector, gatekeepers are commonly seen as primary care physicians. Examples include managed care in the United States and general practitioners in the UK National Health Service. For long-term care, several developed countries have gatekeepers in mechanisms of case management to guide the elderly who return home after hospitalization, as reviewed by OECD (2005) and Johri et al. (2003). However, gatekeepers are not a part of social insurance programs for long-term care in the Netherlands, Germany ${ }^{1}$, or the recent program in Korea.

Our study offers a unique perspective on the provision of formal elderly care using gatekeepers through the radical social insurance experience of Japan, who face the fastest aging population globally. Considering the nature of required treatments, gatekeepers can be a suitable tool in elderly care. Unlike medical care, in which one hospital or one doctor is responsible for comprehensive care, long-term care requires various services. Thus, gatekeepers can promote efficiency in matching the care needs of the elderly with service providers, as opposed to the elderly contacting and visiting specific service providers directly. In addition to supply-side variety, consumer heterogeneity complicates the provision of elderly care. In long-term care, appropriate treatments are more patient-specific than in the case of medical care. Therefore, in addition to health status, other consumer characteristics need to be considered when constructing a suitable combination of services. For example, the presence of a coresident family caregiver can decrease the demand for home care. In this case, a day care service might be preferred to offer respite for the caregiver.

Additionally, the difficulty of collecting information on the quality of providers means the costs to consumers of finding suitable providers might be larger in elderly care than they are in medical care. In many cases, new consumers do not have sufficient time to choose preferable providers, especially when the demand for long-term care emerges after a sudden illness such as a heart attack or accidental injury. Furthermore, it is not always helpful to gather information on a specific service sector, because what constitutes

\footnotetext{
${ }^{1}$ In 2008, the German program introduced a sector that is translated as Information center (Rothgang, 2010). According to Campbell et al. (2010), this sector plays a role similar to that of care managers and was "partly based on Japan's experience."
} 
a suitable service frequently changes in response to the unstable health status of the elderly.

The gatekeeper mechanism also has an advantage specific to the Japanese LTCI. The LTCI is relatively new and, thus, there is limited available information on it. Furthermore, the LTCI program is amended every three years. The program is complicated and unstable rules have widened the knowledge gap between consumers and care providers, making it more costly to collect information on the LTCI than in the case of ordinary doctor-patient relationships.

In spite of the desirable aspects outlined above, the asymmetric information problem common to the healthcare sector creates several difficulties when designing a care management mechanism. As knowledge on health status is available only to a doctor, healthcare sector often suffers from the expert agency problem. Furthermore, in health economics, there is a double agency problem, which arises between patients and doctors and also between doctors and insurers, as described by Blomqvist (1991). For example, a doctor who is the perfect agent for a patient exaggerates the care he or she provides to socially wasteful levels. On the other hand, if a doctor is a perfect agent for the insurer, treatment levels must be lower than the social optimum.

These expert agency and double agency problems are also present in the LTCI. For the expert agency problem, care managers are experts in diagnosing someone's health status and in the complicated LTCI mechanism. Thus, the care management mechanism is more susceptible to the information problem. In the case of the double agency problem, the situation is the same as that of medical gatekeepers in countries with public health insurance, because the LTCI is the national program.

Such agency problems might be addressed by designing an incentive mechanism that ensures the socially desirable behavior of agents. There are three major payment mechanisms, namely a salary, capitation, and a fee-for-service. While researchers have examined how to combine these three mechanisms to handle the agency problem, as summarized by McGuire (2000), each of them is capable of causing the agency problem. In particular, the fee-for-service payment is known to cause supplier-induced demand, which has been actively studied in health economics. This problem creates a financial burden for social insurance. Demand inducement by suppliers can occur in the market under information asymmetry when suppliers, typically physicians, only have access to information about the quality of services. When suppliers face an event, such as accelerated competition, to shift the supply curve upward, they control consumer demand to maintain their income at the level it was prior to the supply-side shock.

In terms of the incentive mechanism, the care management mechanism in the LTCI is operated in a peculiar way. While providers of care services are rewarded by a feefor-service payment, care managers are paid by capitation. Specifically, care managers receive a fixed reward for each care plan, regardless of the content of the plan. The capitation payment is considered cost-effective for insurers, because it does not produce an incentive for care managers to exaggerate demand. However, under the current LTCI, the total reward of care managers, even at best, does not seem sufficient to specialize in care management. Thus, many care managers work for firms who also operate within the service provision sector. These care managers are likely to refer consumers to their 
associated providers. If the choice of a care manager directly corresponds to the choice of providers, this mechanism is equivalent to a direct service supplied by specialists. In this case, these care managers might be rewarded by fee-for-service payments, as a part of service providers.

As a result, there are broadly two categories of care managers, in terms of their payment. The first category consists of independent care managers who work only as care managers and are rewarded by a capitation payment. The second category consists of dependent care managers who work for firms that also operate as service providers. The dependent care managers might be compensated by a fee-for-services payment, which violates the neutrality of care managers. This, in turn, may cause a supplier-induced demand and financial pressure on the LTCI budget. As a result, in 2006, the government introduced a penalty for care management offices that refer too many consumers to a specific provider.

This study examines whether supplier-induced demand exists in care management to determine whether care managers are actually compensated via a fee-for-service payment. Our empirical analysis is conducted using a pooled panel data set from the regional data of local insurers. We employ pooled regression analysis on the regional density of care managers for dependent variables that measure regional consumer demand for care services. The positive correlation between these elements, if any, might imply the existence of supplier-induced demand. Similar analyses based on regional data were used in classic studies such as Fucks (1978) and Cromwell and Mitchell (1986) to detect supplier-induced demand.

However, in the empirical literature on supplier-induced demand, the use of regional data has been criticized from the perspective of identification by studies such as Dranove and Wehner (1994). This problem with regional data prompted recent studies such as Gruber and Owings (1996), SØrensen and Grytten (1999), and Iversen and Lurås (2000) to use microdata of individual suppliers or consumers.

Owing to limited data availability, we utilize regional data and carefully handle the following two problems to justify our analysis. The first problem is that the regional data ignore price heterogeneity. Our analysis of Japanese care managers is free from this problem since price heterogeneity is controlled by the LTCI, which formulates uniform pricing for care services. The second problem is a danger of simultaneity bias caused by the possibility of more care managers being attracted to markets that offer higher profits. We deal with this simultaneity problem via the instrumental variable method.

Our empirical analysis shows that induced demand by care managers may exist, particular in the case of day care services. This result can be naturally interpreted that demand in more beneficial categories becomes a target of demand inducement. Furthermore, we separately analyze independent and dependent care managers, and find a large positive correlation between the density of dependent care managers and consumer demand. This result might provide further evidence of care manager-induced demand.

We also find that care managers in nonprofit organizations might induce more demand than care managers in for-profit firms do. This result might imply crowding out by nonprofit firms who began operating in the market before the establishment of the LTCI. Furthermore, we find that the penalty to encourage care manager neutrality might not 
be effective.

Based on our results, we conduct the following quantitative analysis. An increase in the number of care managers per 1,000 population might generate approximately JPY 7,368 additional demand per year, which implies an additional JPY 736 as out-of-pocket expenses. Although this amount may not be significant to an individual consumer, it can produce JPY 68 billion, or approximately USD 0.68 billion, in excess aggregate costs for the social insurance budget. Given the potentially large impact of care manager induced demand, the independence of care managers deserves more attention to ease this financial burden.

Our study contributes to current literature on the role of gatekeepers. A decade since a classic survey by Scott (2000) noted the lack of studies on gatekeepers in health economics, many theoretical studies have been conducted, such as Garicía-Marinñoso and Jelovac (2003), Malcomson (2004), Blomqvist and Léger (2005), Brekke et al. (2007), González (2010), and Allard et al. (2011). Although these works have not reached a consensus on whether gatekeepers are preferable from an economic perspective, many studies agree on the difficulty of designing an efficient incentive mechanism for gatekeepers. In terms of empirical literature, Mitchell and Sass (1995) detected demand inducement caused by the vertical integration of primary care and specialized hospitals, which might indicate the loss of efficiency of gatekeepers. Croxson et al. (2001) reported that the introduction of a new payment mechanism for gatekeepers in the UK changed their behavior. On the other hand, Grytten and SØrensen (2001) compared the behavior of salaried and fee-for-service physicians in Norway, and did not find demand inducement, even for the fee-for-service physicians. Thus, there is no consensus in the findings of empirical studies either.

Our study makes a unique contribution to the existing literature and provides a more focused view on the role of gatekeepers. Previous studies have focused on primary care physicians, who themselves provide patient care. However, care managers are pure gatekeepers in that they do not provide a care service themselves. Instead, they specialize in referring consumers to service providers. To the best of our knowledge, this category of pure gatekeepers is not seen in any other field of health economics. Therefore, we believe our study will provide a clearer understanding of the role of gatekeepers.

This study also contributes to the literature on empirical studies of the Japanese LTCI. The effects of the LTCI has been analyzed from various perspectives. For example, Iwamoto et al. (2010) discussed the welfare effects of the LTCI in terms of the household consumption, while Sugawara and Nakamura (2014) considered effects on the female labor supply. On the supplier-induced demand in the LTCI, Yamauchi (2004) and Yuda (2005) analyzed home care providers based on regional cross-section data, and found contradicting results. The former study detected induced demand, while the latter did not. In addition, Noguchi and Shimizutani (2009) analyzed home care providers using microdata and did not find evidence of demand inducement. However, these studies did not consider the endogeneity problem between supplier density and demand for care services. Thus, our study should provide more robust empirical results.

The remainder of this paper is organized as follows. In Section 2, we provide a brief review of the LTCI and the role of care managers. Section 3 presents our econometric 
framework and the dataset, and Section 4 summarizes our empirical findings. Finally, Section 5 concludes the paper.

\section{Care manager incentives in the Japanese $\mathrm{LTCl}$ program}

\subsection{The role of care managers}

The Japanese LTCI is a market-oriented program geared toward satisfying the growing demand for elderly care. Unlike the programs in Germany, the LTCI does not allow for direct cash transfers to the elderly or caregivers. Instead, the coverage is limited only to care services purchased through the formal market. This property of the LTCI has created a large demand for long-term care services, enabling the rapid growth of the care industry ${ }^{2}$.

The LTCI originally covered the institutional care and home-based care sectors, and was subsequently amended every three years. The Appendix summarizes the previous amendments. The 2006 amendment was a major reform and created two additional sectors for the LTCI. The first is the preventive care sector for the elderly with lighter care needs, and the second is community-based care in which local authorities have more administrative power than in other sectors. Considering that most developed countries only have a major formal market for permanent institutional care, the wide variety of available services is a unique property of Japanese elderly care.

To guide consumers in this new and complicated market, the LTCI introduced the care manager as an intermediary between consumers and service providers. Care managers offer a menu of services called the care $\operatorname{plan}^{3}$ to each consumer. The care plan describes the amount of services and the firms who provide the services. To create an appropriate care plan, care managers are required to make a careful prior assessment and hold a meeting with providers. After creating a care plan, care managers should visit each consumer at least once a month to monitor the plan and to update it if necessary.

The care managers are associated with three workplaces, namely the preventive care, institutional care, and home-based care sectors. Care managers in the preventive care sector belong to the community comprehensive care center (Chiiki Houkatsu Shien Center), which is operated by either a local authority or a corporation entrusted by the authority. In the home-based care sector, care managers work for the home-based care management office (Kyotaku Kaigo Shien Jigyousho). This study concentrates on care managers in the home-based care sector. The number of active establishments and active care managers in home-based care management increased from 17,176 and 27,542 in 2000 to 27,961 and 65,178 in 2009 , respectively. ${ }^{4}$

\footnotetext{
${ }^{2}$ Ikegami and Campbell (2000) describe the original LTCI and Tsutsui and Muramatsu (2007) provide extensive information about the 2006 amendment. Tamiya et al. (2011) presents a comprehensive review of the current LTCI. For English translations of Japanese legal terms about the LTCI, we referred to Sumii and Sawada (2012).

${ }^{3}$ Although anyone can create a care plan, including consumers, mostly care plans are made by care managers.

${ }^{4}$ These figures are taken from publicly accessible aggregate data of the Survey on Institutions and
} 
Home-based care management offices can be operated either by for-profit or nonprofit firms. Before the establishment of the LTCI, only nonprofit firms were allowed to operate in most parts of the elderly care sectors. The LTCI allowed for-profit firms to enter the market for the first time to meet the rapid growth in demand. However, the existence of an unfair environment for competition between new for-profit firms and incumbent nonprofit firms has often been reported. ${ }^{5}$

The main services in the home-based care sector are home care (Houmon Kaigo) and day care services (Tsuusho Kaigo). Home care is a service in which paid caregivers visit the elderly and provide long-term care at home. The day care service provides long-term care to the elderly in a facility during the daytime, without overnight stay. Unlike institutional care, not requiring beds promotes offering care to more consumers at the same time. This property indicates that day care services can benefit from good management. Detailed information on the management practice of these services can be found in the Survey of Long-Term Care Management (Kaigo Jigyou Keiei Jittai Chousa), which is a sampling survey for establishments conducted by the Japan Ministry of Health, Labour, and Welfare (JMHLW) every three years. The results for 2005 and 2008 show that the average profit of home care establishments is approximately zero, while day care services rewards are $7 \%$ greater than cost.

In the 2006 amendment, two policies were assigned to encourage substituting homebased care for costly institutional care. The first is the Regulation of Volume (Souryou Kisei), which prohibits creating new institutions. ${ }^{6}$ This is not a formal rule. Rather, it was an announcement made by the government to each insurer, a regional unit of authority to operate the LTCI. The second policy is assigning room fees and board expenses for institutional care, which were previously covered by the LTCI, as out-ofpocket expenses for institutional care. These policies made day care services increasingly popular as a convenient alternative to institutional care.

\subsection{Incentives for care managers}

The LTCI is a social insurance program with universal coverage. Whenever long-term care is needed, the elderly person requests an assessment to obtain an LTCI classification level. This classification level is assigned based on several items, including activities of daily living (ADL) and instrumental activities of daily living (IADL). Before the 2006 amendment, there were six classification levels, namely assistance-required (AR) and care-required (CR) 1 to 5, where the latter levels cover costs for more intensive care. Then, the 2006 reform redefined the lighter levels, changing AR and CR 1 to AR 1 and 2 and CR 1. The AR 1 and 2 levels were subsequently distributed to the new preventive care sector.

\footnotetext{
Establishments for Long-Term Care.

${ }^{5}$ For example, Suzuki (2002) detected a nonprofit premium in consumers' choice for home case service providers and interpreted this to be the result of an unequal environment for nonprofit and for-profit firms.

${ }^{6}$ See Sugawara (2015) for details of the Regulation of Volume and its impact on the nursing home industry.
} 
The assigned classification level prescribes a set of available care services and an upper bound on how much insurance will pay out. The upper bounds have not changed since the launch of the LTCI. Out-of-pocket expenses are $10 \%$ of the costs within the bound, with the remainder covered by the LTCI. After obtaining the classification level, the consumer chooses a care manager for a care plan. The consumer can choose any care manager, and can also switch to a different care manager at any time.

Service providers are rewarded via a fee-for-service payment. However, the prices of services are controlled by the government. Similar to medical care in Japan, the LTCI adopts a detailed remuneration points system to determine rewards for services. As a result, most elderly care services are provided at a fixed price in Japan. The only element that produces a price difference is the regional variation in the exchange rates between remuneration points and money $^{7}$. In this study, we use remuneration points to measure consumer demand.

Care management is paid via capitation. In other words, regardless of the contents of a care plan, care managers receive a fixed reward for each plan, with a few exceptions in the form of special bonuses. Ignoring bonuses, care managers today receive approximately JPY 10,000 per consumer per month. As summarized in Appendix, care managers have been limited to seeing at most 39 consumers since the 2006 LTCI amendment. Thus, the total reward of a care manager, at best, and without bonuses, is less than JPY 400,000 per month. This reward does not seem sufficient to operate a business that specializes in care management.

Consequently, many care managers work for firms that also operate within the service provision sectors. These care managers are likely to refer consumers to the associated providers. If the choice of a care manager directly corresponds with a choice of providers, this mechanism is equivalent to a direct service supplied by providers. Therefore, care managers might be compensated by a fee-for-service payment as part of their work for the service providers if their neutrality cannot be guaranteed.

The LTCI amendments changed the remuneration points for home-based care management, as summarized in the Appendix. In particular, two important policies were introduced to encourage the neutrality of care managers. The first policy provides a reward in the form of an additional bonus for care plans in difficult situations. For example, a care plan for an elderly person who has just been discharged from hospital receives a bonus. In 2009, this bonus consisted of $12 \%$ of the total payment for homebased care management ${ }^{8}$. Because, the impact of the bonus seems minor, we ignore it in this study.

The second policy is a penalty if a care management office employs a specific provider in more than $90 \%$ of their care plans. The rate is not measured based on the amount of service provision, but rather on whether the provider is employed. However, under this rule, dependent care managers can still benefit a specific provider while avoiding the penalty.

\footnotetext{
${ }^{7}$ Originally, there was no regional difference in the exchange rate, and all points were exchanged for JPY 10. However, regional differences were introduced in 2003.

${ }^{8}$ The figure is taken from the Survey of Long-Term Care Benefit Expenditures (Kaigo Kyuufu-hi Jittai Chousa)
} 
For example, consider the following imaginary care plans produced by a care management office. The office has two customers, elders 1 and 2. Elder 1 needs 99 hours of home care per month, while elder 2 requires 1 hour of home care. There are two home care providers, $\mathrm{A}$ and $\mathrm{B}$, in this area. The imaginary care plan for elder 1 distributes all 99 hours to provider A, while the care plan for elder 2 distributes 1 hour to provider B. In this case, provider A occupies $99 \%$ of the demand. However, the penalty is not imposed on the care management office because providers A and B are used in equal $(50 \%)$ measure, with each being allocated in one care plan.

\section{Empirical framework}

\subsection{Econometric model}

Our empirical analysis is based on regional pooled panel data. The unit of the regions is the insurer, who is a local authority to exercise the administrative power of the LTCI program. Insurers are mainly individual municipalities, but some municipalities collaborate with others to administer the LTCI program. There are two legal forms of collaboration, the wide-area unions (Koiki Rengo) and the partial-affairs associations (Ichibu Jimu Kumiai). These two forms essentially have the same set of functions, thus, we do not pursue their difference in this study. ${ }^{9}$

The Japanese municipalities experienced active mergers during the first half of the 2000s. The number of municipalities decreased from 3,229 on November 1, 2000, to 1,727 on April 1, 2010. ${ }^{10}$ Owing to this political change, we have an unbalanced panel dataset of insurers. In other words, the sample consists of $N$ insurers, where the $i$ th insurer was observed for $T_{i}$ years.

For this sample, we employ a simple linear regression analysis using the pooled data. The dependent variable is the number of remuneration points for home-based care per care plan. The remuneration points measure the quantity of utilized services. Since the prices of formal care services are fixed by the LTCI, the points correspond with consumer demand. We adopt observable characteristics of regions as explanatory variables. Our main interest is the effect of care manager density on demand. If this variable has a positive coefficient for consumer demand, this may imply that care managers induce demand.

Although we have panel data on insurers, we utilize a pooled regression analysis. As mentioned in a later section, our estimation utilizes data of only eight years. Furthermore, owing to the considerable changes in the municipalities in the 2000s, many insurers only appear for short periods. After considering these factors, we opted not to include regional fixed effects to avoid the problem of overfitting. Instead, we adopt

\footnotetext{
${ }^{9}$ Jacobs (2004) provided a detailed description of multi-municipal collaboration in Japan. We have referred to CLAIR and GRIPS (2010) for the English terms "wide-area union" and "partial-affairs association."

${ }^{10}$ Jacobs (2011) provided details of Japanese local authorities and their history, including the recent mergers.
} 
cluster standard errors to control for insurer-specific elements in the pooled regression framework.

There may be simultaneity between the dependent variable and the care manager density, because highly profitable areas might be attractive to care managers. To eliminate a possible simultaneity bias, we use the instrumental variable method, where our choice of instruments are described in Section 3.2.3.

\subsection{Data}

\subsubsection{Definition of the sample}

To construct regional panel data of insurers, we carefully define the sample unit to handle municipality mergers. We need to trace several important variables from the Census, ${ }^{11}$ which is held each five years, for new municipalities established by mergers. ${ }^{12}$ We define the new municipalities even if they share the same name as a municipality before the merger. In addition, if municipalities experienced a change without a physical difference, such as a change in name, we do not treat them as new municipalities. For multimunicipal collaborations, ${ }^{13}$ we have created new samples if municipalities changed in terms of composition. However, if mergers occurred between constituent municipalities, we do not treat the collaboration as a new sample.

We define the insurer as the unit where the demand inducement can occur. A potential weakness of our study is the possibility of a border crossing problem. This problem is explained by Dranove and Wehner (1994) as a weakness emerging from the use of regional data to detect supplier-induced demand. Home-based care management offices and care providers must declare their area of operation, which is not limited to the insurer they are located within. It is relatively common for home-based care management offices to include multiple insurers in their operational area. Further, when there is a request from outside the operation area, the home-based care management office can accept the offer and pay additional transfer fees. Therefore, there may be consumers who access the service from outside the insurer. Since we do not have access to information on the operation areas, this problem remains unsolved in this study.

\footnotetext{
${ }^{11}$ Census information for Miyake Village in the Tokyo prefecture could not be obtained for 2000, because all dwellers were evacuated following a volcanic eruption. Thus, we eliminate this insurer until the next census round in 2005 .

${ }^{12}$ The merger records can be obtained from the homepage of the Japan Ministry of Internal Affairs and Communications (http://www.soumu.go.jp/gapei/gapei.html), accessed on October 6, 2013. Kamikuisshiki village in the Yamanashi prefecture experienced both a split and a merger in 2006. Then, we eliminate the municipalities of Kofu city and Fujikawaguchiko town, which incorporated part of the former Kamikuisshiki village. All other mergers are simple aggregations of areas and we can trace their pre-merger status.

${ }^{13}$ Information about changes in the composition of municipalities for multi-municipal collaboration is taken from the web page of the Japan Ministry of Internal Affairs and Communications (http://www.soumu.go.jp/kouiki/kouiki1.html) and WAM NET (http://www.wam.go.jp/ content/wamnet/pcpub/top/), which is a government-owned web page disclosing information about medical and long-term care. These websites were accessed October 6, 2013.
} 


\subsubsection{Definition of dependent variables}

Our dependent variable is the number of remuneration points for home-based care per care plan. The numerator is the number of remuneration points for home-based care services, while the denominator is the number of care plans created by home-based care management offices. Both numbers are taken from the Annual Report on LTCI (Kaigo Hoken Jigyou Joukyou Houkoku). Since the Annual Report on LTCI provides only prefecture-level information on the number of care plans in 2000, we utilize data from 2001.

The remuneration points are annual aggregate values for the entire home-based care sector minus the points allocated for home-based care management. In this analysis, we concentrate on the remuneration points for heavier care requirement, namely classification levels CR 3 or above ${ }^{14}$. We put this restriction to avoid complication from the 2006 amendment which changed the categories for lighter care needs, as described in Section 2.2. Furthermore, this restriction can clarify the care manager-induced demand, if any, because the heavier classification levels have more generous coverage which might produce enough room to induce consumer demand. The number of care plans is measured as the annual number of recipients of home-based care management with the heavier care requirement. This number includes not only new care plans, but also the monitoring of ongoing care plans and bonuses.

Furthermore, the dependent variable might be distorted owing to the complicated legal system in the home-based care sector. For example, care plans for dementia group homes and private nursing homes are made by care managers belonging to these services, and not by home-based care management offices, even though those institutions are categorized in the home-based care sector. However, the Annual Report on LTCI does not provide information on individual services for several years.

To make robust inference to policy changes, we further construct dependent variables with respect to demand for the home care and day care services, which are two major services in the home-based care sector. According to the Survey of Long-Term Care Benefit Expenditures, home care and day care services are utilized by more than $30 \%$ of elders with all classification level during the research periods. However, Nakamura and Sugawara (2016) showed that there are distinct patterns of utilization for these services depending on household structures of consumers. Thus, we need to control demographic factors carefully to capture the demand patterns for these services.

\subsubsection{Definition of care manager densities and the instrument}

Our main explanatory variable is the care manager density for each insurer. We define this variable as the number of care managers per 1,000 population. The number of care managers for home-based care management offices in each region is calculated using microdata of establishments from the Survey on Institutions and Establishments for

\footnotetext{
${ }^{14}$ We choose this definition of the heavier care requirement based on a recommendation of a former member of the governmental committee to formulate the classification level. According to this person, our definition is most likely to be consistent to changes from the LTCI amendments
} 
Long-Term Care (Kaigo Sabisu Sisetsu Jigyousho Chousa). This is an annual survey conducted by the JMHLW of all formal care service establishments. Since we have access to data until the 2009 round of this survey, our analysis uses data from 2001 to 2009. Furthermore, our sample starts from 2002, because we need the one-year lagged value of the care manager density as an instrument.

The number of care managers in each establishment is calculated as the number of full-time workers. Part-time workers and workers who are also engaged in other jobs are counted as "one full-time worker" if the sum of their working hours reaches the scheduled working hours of full-time workers in each establishment. Several offices also operate as community comprehensive care centers and there can be an overlap in care managers for home-based care and preventive care. In the Survey on Institutions and Establishments for Long-Term Care, such an overlap is separated in the definition of the variables and does not provide a measurement error.

Owing to the availability of microdata, we also employ two additional analyses using alternative definitions of care manager densities. First, we analyze densities of dependent and independent care managers. An independent office is defined as an office that does not include a service-provision sector for home-based services. This group also includes multifunctional long-term care in a small group home. This belongs to the community care sector, but is referred by care plans of home-based care management. However, in the home-based care sector, we exclude two services in the definition of independent care managers. First, the visiting nurse service is eliminated because the Survey on Institutions and Establishments for Long-Term Care in 2003 does not ask the home care management sectors about joint operations in this sector. Second, we do not include care in private nursing homes. The private nursing home sector may be motivated to attract home care consumers as future institutional care consumers. However, this motivation does not induce demand for home-based care services.

Second, we analyze the densities of care managers who work for for-profit and nonprofit firms. This analysis considers the importance of firm ownership, which is another arguable topic in the LTCI as mentioned in Section 2.1.

To control the endogeneity between care manager densities and the long-term care demand, we utilize two instruments. The first instrument is the lagged value of the care manager density, which is justified as follows. For the exogeneity on long-term care demand per care plan, there is no clear path that the lagged care manager density affects the current demand after controlling the current care manager density. On the other hand, it is not easy to change locations for care management offices, owing to sunk costs. Thus, in a natural setting, there should be a correlation between current and previous care manager densities. For the analysis on alternative definitions of care manager densities, we construct the corresponding lagged variables for the instrument by care manager types separately.

The second instrument is the non-user ratio, which is defined as the ratio of the number of elders who do not use the home-based care services to the number of whole elders. The non-users contain both healthy elders and disabled elders without utilization of home-based care. As mentioned later, we adopt ratios of the elderly in each LTCI classification level as explanatory variables. After controlling characteristics of current 
consumers by these variables, there is no clear path for the number of non-customers to affect the per capita demand from customers. On the other hand, the non-user ratio can be an important factor for prediction of the future market structure which might have an influence on the location choice of care management offices. Thus, this variable is likely to correlate with the care manager density.

\subsubsection{Definition of other explanatory variables}

In addition to the care manager density, we also adopt the following explanatory variables. Three variables are adopted to measure the general market size of insurers. The first variable is regional population density. Here, we take the regional population from the Vital Statistics as the numerator of population density. For the denominator, data on regional areas are taken from annual reports of the Land Survey of Prefectures Shi, $\mathrm{Ku}$, Machi, and Mura (Zenkoku Todoufuken Shikuchouson Betsu Menseki Shirabe). This variable can also measure the economic activity of the regions.

The other two variables on market size measure the potential size of the elderly care sector and are taken from the Census. The first variable is the ratio of households that consist only of a single elderly person to other households that have at least one elderly member. An elderly person is defined as a person aged 65 or older. The second variable is the ratio of households that consist only of an elderly couple to other households that have at least one elderly member. An elderly couple is defined as a husband aged 65 or more and a wife aged 60 or more. These variables are referred to as the single elderly household ratio and the elderly couple household ratio, respectively. In addition to the role to measure the market size, these variables also reflect the family structure of regions. The family structure might affect the demand for formal long-term care, because provision of informal care by family members can substitute the formal care, as suggested by van Houtven and Norton (2004) .

The economic status of insurers is incorporated into our analysis by two variables. The first variable is the municipality local tax (Shi Cho Son Min Zei), which is taken from the Annual Statistics on Municipal Tax (Shi Cho Son Zei Kazei Joukyou Tou no Shirabe). This tax includes the fixed asset tax and inhabitant taxes for individuals, corporates, and small components ${ }^{15}$. We cannot obtain separate detailed tax components before 2007 and, thus, use the aggregate value. We divide this variable by the regional population to control for different areal scales and take a logarithm. We also adopt the squared term for the logarithm value in the empirical analysis.

The second variable on economic status is the rate of workers in the primary industry, which is taken from the Census. Because there is a circumstance of mandatory retirement in Japan, most of the former employed workers do not have income sources other than the public pension in their later lives. On the other hand, because self-employed workers are free from the mandatory retirement, they might have their own income source which can create a different economic perspective. We do not have access to statistics about general self-employed workers in the municipality level. However, using the figure for the primary industry, we can control a major category in the self employment in Japan.

\footnotetext{
${ }^{15}$ See Harada (2009) for details on Japanese local taxation.
} 
To control for the quality differences in required care services, we analyze the distribution of disabilities among the elderly for each insurer. This element is measured by ratios of the elderly in each LTCI classification level to the elderly who have any LTCI classification in the category of the heavier care requirement. Since the sum of the ratios for all levels takes unity, we eliminate the ratio of CR3 to avoid multicollinearity.

As mentioned in the previous section, we do not control for the fixed effects of insurers because of the lack of observation periods. Instead, we include year dummies to handle trend components. Additionally, to incorporate the distinction between administrative units, we include a dummy explanatory variable that takes unity if the insurer is a multi-municipal collaboration. Since we do not have an intuition for the effects of year dummies and the administrative units, we do not provide an interpretation for them in the following empirical analysis.

\subsubsection{Descriptive statistics}

\section{Table 1 here}

Table 1 shows the descriptive statistics. Columns (1), (2), and (3) show the sample means and standard deviations for variables of all periods between 2002 and 2009, the periods before the 2006 amendment, and the periods after the 2006 amendment, respectively. For the dependent variables, the remuneration points per care plan for the heavier care requirement drastically increased after 2006. This is a natural consequence of the diffusion of the formal elderly care service market, which appeared after the launch of the LTCI. Furthermore, home care and day care services occupied about half of home-based care plans for the heavier care requirements.

Care manager densities per 1,000 population before and after the 2006 amendment increased from 0.482 to 0.613 . In addition, the average number of home-based care management offices increased from 8.527 to 14.25. This is further evidence of the ongoing diffusion of the LTCI over this period. Among care managers, independent care managers comprised about one third.

Among the independent variables, the population density has a wide range of values because of the disparity of municipality sizes. To obtain a stable coefficient estimate, we utilize a logarithm value in our estimation.

\section{Estimation result}

\subsection{Basic analysis to detect the scope of demand inducement}

\section{Table 2 here}

We begin our empirical analysis by detecting which sector is exposed to demand inducement by care managers, if any. Table 2 shows the estimation results of the baseline regression analysis for the remuneration points per care plan. Columns (1), (2), and (3) report the estimated coefficients and their cluster standard errors. Here, the dependent variables are the remuneration points for care plans for the elderly with the heavier care 
requirements, home care plans for the elderly with the heavier care requirements, and day care plans for the elderly with the heavier care requirements, respectively.

For our main purpose of estimation, the significantly positive coefficient of the care manager density in (1) can imply the existence of care manager-induced demand. In Columns (2) and (3), we clearly see that demand inducement might occur only in day care services. The low profitability means home care might not be an attractive source for demand inducement. In addition, because home care is frequently provided within view of family members, it is difficult to induce unnecessary demand. On the other hand, the day care service is relatively profitable and is provided out of sight of family members. Considering these factors, our finding fits our intuitive understanding.

For the other explanatory variables, the population density positively affects consumer demand for home-based care. We interpret this as follows. In more urbanized areas, people have more opportunities to obtain a better job. When there is a need of longterm care for coresident family members, workers who are satisfied with their job are likely to outsource their care requirements to formal sectors. ${ }^{16}$ Thus, formal care through the market dominates informal family care for regions with an active economy. This effect causes the correlation between population density and per capita consumer demand for formal care.

The ratios of the elderly in each LTCI classification level show distinctive results on care plans for the day care service and the other categories. For categories other than day care, the ratios with the heavier care levels generally show larger positive effects on demand relative to the ratios for the lighter care levels. In contrast, with regard to demand for the day care service, the ratios of the elderly with the heavier care requirements show larger negative effects. These results can be interpreted as follows. For home care or other home-based care than the day care service, required services increase proportionally to the level of disability of the elderly. Besides, for day care, the demand tends to be decided by caregivers who allocate their time also to activities other than care, rather than by the elderly. Thus, the disability level is not the main determinant of demand. Furthermore, for those who are very disabled, it is difficult to stay at home without devoted caregivers. Given devoted caregivers, demand for day care can be small. On the other hand, as the disability becomes severer, demand for the other services than day care might increase. Thus, demand for day care may relatively decrease with an increase in the disability level.

The single elderly household ratio has a positive effect on home care demand, as shown in Column (2), and a negative effect on day care service demand, as shown in Column (3). This effect may be the result of the existence or non-existence of a coresident caregiver. Without a coresident caregiver, home care is the main service among all other home-based elderly care. In contrast, if a coresident caregiver is present, demand for the day care service provides respite for the caregiver. Single elderly households do not have a coresident caregiver and are more likely to use home care than a day care service.

\footnotetext{
${ }^{16}$ Sugawara and Nakamura (2014) showed a consistent result for this explanation and noted that female regular workers increased their working hours, while non-regular workers decreased their working hours when their coresident elderly person required long-term care.
} 
Therefore, the signs of this coefficient are a natural result.

The elderly couple household ratio has significantly negative effects on demand for both the home care and day care services. For such households, it becomes difficult for one caregiver to support the other severely disabled elder. These households are likely to utilize institutional care instead of home-based care and, thus, become single elderly person households. Therefore, demand for home-based care in households that still consist of only an elderly couple might concentrate on care for lighter needs.

The per-capita municipality tax and its squared term have significant estimates of the coefficients for day care services. Considering the shape of the quadratic functions, we find positive impacts of this variable for day care services. To see this, the $1 \%$ percentile for the $\log$ of the per-capita municipality tax is 48.888 . In addition, the remuneration points per day care plan comprise a convex function with a minimum at approximately $2,586 /(2 \times 311.8)=4.168$, which is below the $1 \%$ percentile. Therefore, as the percapita municipality tax increases, its positive impact generally increases for the day care service. This result can be justified similarly to that of the population density from the perspective of an active economy.

The ratio of workers in the primary industry has a significantly positive coefficient in (1) and not significant but positive in (2) and (3). Thus, in addition to the general local economy, economic situations of elders are influential as a determinant of the per capita long term care demand.

\subsection{Validity of instruments}

In Table 2, to check validity of our instruments, we conduct tests on exogeneity in the second stage and significance in the first stage. For the second stage exogeneity, we conduct the overidentification test using Hansen's J statistics. The test statistics are not significant in $10 \%$ level, which support the exogeneity of our instruments, except for the home care sector.

For the home care sector, we then adopt another inference scheme which includes only the lagged care manager density as an instrument. Because the overidentification test does not work for the inference with only one instrument, we utilize an alternative test which was proposed by Dranove and Wehner (1994) and applied by several studies on the supplier-induced demand such as Grytten and SØrensen (2008). The test statistics is a correlation between the instrument and OLS residuals for the second stage. In the Dranove and Wehner test, the null hypothesis is that the correlation is zero. This test can provide a supporting evidence for the exogeneity of the instrument if the null hypothesis is not rejected. In our inference for the home care sector in (2), the test statistics is not significantly different from zero in $10 \%$ level, which supports the exogeneity of the lagged care manager density in the second stage.

We also test the significance of our instruments in the first stage. In Table 2, we present the coefficients and standard errors for each instrument and the joint test that the coefficients for our instruments are both zero in the first stage regression. All the results show the significance of our instruments in the first stage. For the sign of each instrument, the lagged care manager density has a significantly positive coefficient which 
indicates that the care management offices cannot change their locations immediately, as a natural implication of the existence of sunk costs.

On the other hand, the non-user ratio has a significantly negative coefficient. This result can be justified if a high non-user ratio can be related to a systematic mechanism to reduce a usage of the LTCI services and thus creates a negative prediction for the future market growth. The examples of such a mechanism are general health status of elders, informal community networks for mutual help or a local non-LTCI polity for elderly care. Because our data are not rich enough to discuss details on a underlying mechanism, this topic is beyond the scope of this study and remained as a future task.

The result that the non-user elder ratio is not exogenous for the home care demand can be interpreted as follows. The non-user ratio is related to the density of customers among the area. If these disabled elders lived sparsely, visiting their homes are costful. Such a difficulty can affect the operation of the home care sector. This result indicates a systematic difference between demands for home care and day care services.

\subsection{Detailed analysis on care plans of day care for elders with heavier care requirements}

Table 3 here

Next, we pursue a detailed analysis of care plans for day care among the elderly with the heavier care requirements. Table 3 decomposes the baseline results for day care for heavier care requirements into more detailed categories. Since we have similar signs for the coefficients of the explanatory variables other than care manager densities, the following analysis interprets the care manager densities only.

Table 3 also reports test statistics to validate our instruments. For the first stage significance, we only report the $F$ statistics for the joint test of all instruments for simplicity, but signs of coefficients for all instruments are same as in Table 2 (1) and significant. Because our inferences in Columns (1) and (2) include multiple endogenous variables, we employ the first-stage regression for each endogenous variable, where the non-user ratio and the lagged density for the corresponding category of care managers are included. As a result, both the first-stage $F$ test and the overidentification test show the validity of our instruments.

Column (1) in Table 3 separately considers care managers who belong to offices that also operate day care services, offices that operate a home-based service other than day care, and are independent. The result shows that care managers who are dependent on a day care service induce more consumer demand. The densities of care managers who are independent and dependent on providers other than day care also have a significantly positive coefficient. However, their values are smaller than the dependent care managers on the day care service.

It is possible to relate the shortage of care managers to the positive effects of the densities of care managers without joint operation with day care. If the number of care managers is much smaller than the appropriate figure, it is difficult for them to spend enough time to provide substantial care plans. On the other hand, in regions with sufficient care manager density, they are free from such a time constraint and can 
create more substantial care plans. In this situation, the care manager density will demonstrate its positive effect, but this is not harmful to the public insurance budget. The situation might be also related to that of SØrensen and Grytten (1999), who did not find physician-induced demand in Norway. They justified their finding using the low supplier density, because the lack of competition eliminates the motivation of physicians to induce demand.

Column (2) in Table 3 separately considers care managers who belong to for-profit and nonprofit firms. While both densities have significantly positive coefficients, the value is larger in the case of nonprofit firms. This result might imply crowding out by nonprofit firms, who operated long-term care services before the establishment of the LTCI. On the other hand, although we abbreviate to report a whole table of estimation results, the first-stage regression shows that the population density has a significantly positive effect on the for-profit care manager density, but a significantly negative effect on the nonprofit care manager density. Combining these estimation results, there might be regional differences in demand-inducement by care managers.

Columns (3) and (4) in Table 3 separately consider the period before and after the 2006 amendment, respectively. The coefficients for the care manager density are not largely different in these two periods. This result implies that the penalty introduced by the 2006 amendment did not effectively prohibit demand inducement. Since the amendment was established for all insurers, we cannot conduct a difference-in-difference analysis to control for chronic changes in unobservable elements around the time of the political change. Thus, a more robust analysis on this topic remains as a future task.

\subsection{Robustness check}

In this subsection, we provide additional analysis for robustness check ${ }^{17}$. The following analysis focuses on care plans for heavier care in the day care services. For simplicity, we unite care managers in the independent offices and dependent offices with on day care in the name of non-dependent care managers.

Our additional analysis is intended to control the possible simultaneity between the care manager densities and the units per care plans. Because our analysis utilizes data during periods of rapid diffusion of the LTCI, there can be positive time-variant, unobservable factors which affect both of these elements. Our analysis so far shows validity of our instrument, which is a supportive evidence that we successfully avoid the endogeneity bias. On the other hand, the consideration of the simultaneity can provide further implications on the possible time trends. For this purpose, we test the existence of positive differences between coefficients for dependent and non-dependent care manager densities.

\footnotetext{
${ }^{17}$ We also conduct additional estimation using a balanced panel dataset of municipalities that did not experience merging during the research period, to control regional fixed effects. However, we obtain unstable coefficient estimates with large standard errors, possibly by two reasons. The first is the shortage of observation periods. Because we only have eight years for observations, the insurerspecific effects might cause overfitting. The second reason is the sample selection. Because the merging of municipalities is generally motivated by economic reasons, our choice of a sample may cause a selection bias.
} 
This test works as the following mechanism. The possible time trend can affect behavior of care managers regardless of their relationship to the service provision sector. On the other hand, as shown in our estimation above, care managers without dependence on the service provision sector is free from the incentive problem of demand inducement. Therefore, if we take the difference between the coefficients for dependent and non-dependent care managers, we can isolate the the effect of incentives on dependent care managers from the possible time-variant factors.

We specifically conduct following two analysis. The first analysis considers overall effects using coefficients for all time periods. The second analysis utilizes cross terms of care manager densities and year dummies and conducts testing for their difference in each year. This second analysis is intended to control for the potential time trend using the advantage of panel data. In the second analysis, we do not adopt the instrumental variable method, because one of our instrument, the lagged care manager density, also works as a dependent variable in this scheme. In other words, the second analysis is specializing in controlling the possible time trends by neglecting the other endogenous factors, if any.

\section{Table 4 here}

Table 4 reports results for estimation and testing. The first row shows results for the first analysis on the overall effects, while the other rows shows results for the second analysis. The difference between coefficients for dependent and non-dependent care manager densities is significantly different from zero for the whole period in the first analysis. On the other hand, the second analysis shows significant differences except for 2006, while the result is different in 2006. The $\chi^{2}$ statistics for the differences take stable values before and after 2006, respectively. On the other hand, the differences are shrinking in recent years, although they are still significantly positive.

These findings imply that the major amendment of the LTCI in 2006 caused a temporary complication of operation for care management and service provision in this year. Furthermore, there exists a shrinking time trend, which is caused by factors that are not directly observable or measurable in our regional data. Therefore, except for the year of transition, our analysis consistently shows existence of supplier induced demand by dependent care managers, even controlling the possible time-trend. Thus, this analysis exhibits the robustness of our findings. On the other hand, what happened in 2006 and what causes the shrinking trend are also important questions, but they are beyond the scope of our research and remained as future tasks with mode detailed data.

\subsection{Impact of care manager-induced demand on the LTCI budget}

Using the baseline result in Column (1) of Table 2, we can consider the quantitative impact of care manager-induced demand. An increase in the number of care managers per 1,000 population causes an increase of 1,357 points per care plan for elders with the heavier care requirements. The annual average induced demand per such a care plan can be calculated as the product of three terms: the regional average number of care managers, the estimated induced remuneration points per care manager, and the exchange rate between remuneration points and money. We ignore the regional difference 
in the exchange rate and use the minimum value for simplicity. Then, we obtain the average induced demand as $0.543 \times 1,357 \times 10 \simeq 7,368$. Thus, induced demand caused the annual cost per care plan for the heavier care requirements to increase by JPY 7,368 , of which JPY 736 formed an out-of-pocket expense.

Individual consumers may find this expense negligible. However, if this cost is levied on many care plans, then on average, this excess payment constitutes a large burden on the social insurance finance. In 2009, there were 9,333,675 care plans made by home-based care management offices for elders with the heavier care requirements. This number is taken from the Annual Report on LTCI. Multiplying this number by the average cost increase caused by induced demand, we obtain a total loss of JPY 68 billion, or approximately USD 0.68 billion per year. As shown in Yong and Saito (2009), the life expectancy with heavy disabilities have been growing in Japan. Thus, this cost due to the care manager-induced demand is likely to increase further in the near future.

Our results also show that independent care managers do not induce demand. Therefore, we claim that care managers remunerated by capitation payments are not harmful to the LTCI budget. Instead, the current mechanism cannot guarantee the neutrality of care managers, which is necessary to financing the social insurance. The problem here is that the reward for care managers is too small to support independent care management.

\section{Conclusion}

This study provided an empirical analysis of care managers, who can be considered as pure gatekeepers, in the Japanese social insurance program for elderly care. The insurance program was originally designed so that care managers would be compensated by capitation payment, which is cost-effective for the insurance budget. However, there is a possibility that care managers are actually rewarded by a fee-for-service payment, which might place a financial burden on insurance via supplier-induced demand. Using regional pooled-panel data of insurers, we empirically found a positive correlation between care manager density and service costs. Our empirical study revealed that care manager-induced demand might exist and imposes a large financial burden on the social insurance program.

The limited availability of data means there are several remaining tasks. First, our empirical study is still exposed to a potential border-crossing problem, because insurers may be too small to capture real market demand. Further analyses using wider regions as a sample unit, such as the secondary medical care area (niji iryou ken), are desirable. As a result of the massive changes to Japanese municipalities in the 2000s, data handling becomes difficult and is beyond the scope of this study.

Second, our approach has a difficulty in that we do not have access to micro data. Due to the data limitation, we utilize a reduced form approach and there is a possibility that our finding does not totally correspond to the supplier induced demand. On the other hand, in our perception, our main contribution is to suggest existence of an problem in the LTCI through empirical analysis using large data from all over Japan. This is a rough but socially important finding which needs to be supplemented by further studies using 
more detailed models and data. In this sense, our study can be a significant starting point for more detailed empirical studies.

For example, microdata on individual care managers or individual care plans, if any, will enable more intensive research on the following two topics. The first is that capitation payment can cause less effort by gatekeepers. This topic is conventionally studied in health economics theories, such as the studies of Malcomson (2004) and Blomqvist and Léger (2005). The second topic is the normative aspect of interpretation. It is possible for induced demand to have positive impacts on the health of consumers, as discussed in Labelle et al. (1994). Since we do not have data for detailed purchase schedules or the health status of individuals, we do not examine these two aspects in this study. These remain as future topics of research.

\section{Acknowledgments}

The authors thank Hidehiko Ichimura, Kensuke Miyazawa, Katsumi Shimotsu, Shuzo Nishimura and seminar participants at the University of Tokyo, Osaka University, JEA and IHEA for helpful discussions and comments. This work is supported by a Grantin-Aid for Scientific Research(B) No. 23330085 and No. 26285066 from the Japanese Ministry of Education, Science, Sports, Culture and Technology and JST CREST. The computational results are obtained by using Stata 13.

\section{References}

M. Allard, I. Jelovac, and P. T. Léger. Treatment and referral decisions under different physician payment mechanisms. Journal of Health Economics, 30:880-893, 2011.

A. Blomqvist. The doctors as double agent: Information asymmetry, health insurance, and medical care. Journal of Health Economics, 10:411-432, 1991.

A. Blomqvist and P. T. Léger. Information asymmetry, insurance, and the decision to hospitalize. Journal of Health Economics, 24:775-793, 2005.

K. R. Brekke, R. Nusheler, and O. R. Straume. Gatekeeping in health care. Journal of Health Economics, 26:149-170, 2007.

J. C. Campbell, N. Ikegami, and M. J. Gibson. Lessons from public long-term care insurance in Germany and Japan. Health Affairs, 29(1):87-95, 2010.

J. Cromwell and J. B. Mitchell. Physician-induced demand for surgery. Journal of Health Economics, 5:293-313, 1986.

B. Croxson, C. Propper, and A. Perkins. Do doctors respond to financial incentives? UK family doctors and the GP fundholder scheme. Journal of Public Economics, 79: 375-398, 2001. 
D. Dranove and P. Wehner. Psysician-induced demand for childbirths. Journal of Health Economics, 13:61-73, 1994.

V. R. Fucks. The supply of surgeons and the demand for operations. Journal of Human Resources, 13:35-56, 1978.

B. Garicía-Marinñoso and I. Jelovac. Gps' payment contracts and their referral practice. Journal of Health Economics, 22:617-635, 2003.

Geospatial Information Authority of Japan (former Geographical Survey Institute), Japan Ministry of Land, Infrastructure, Transport and Tourism (JMLIT). Land Survey of Prefectures, Shi, Ku, Machi and Mura (Zenkoku Todoufuken Shikuchouson Betsu Menseki Shirabe in Japanese). Tokyo, Various years.

P. González. Gatekeeping versus direct-access when patient information matters. Health Economics, 19:730-754, 2010.

J. Gruber and M. Owings. Physician financial incentives and Cesarean section delivery. Rand Journal of Economics, 27(1):99-123, 1996.

J. Grytten and R. SØrensen. Type of contract and supplier-induced demand for primary physicians in Norway. Journal of Health Economics, 20:379-393, 2001.

J. Grytten and R. SØrensen. Busy physicians. Journal of Health Economics, 27:510-518, 2008.

K. Harada. Local taxation in Japan. In Papers on Local Governance System and its Implementation in Selected Fields in Japan. Institute for Comparative Studies in Local Governance and National Graduate Institute for Policy Studies (GRIPS), 2009.

N. Ikegami and J. Campbell. Long-term care insurance comes to Japan. Health Affairs, $19(3): 26-39,2000$.

T. Iversen and H. Lurås. Economic motives and professional norms: the case of general medical practice. Journal of Economic Behavior and Organization, 43:447-470, 2000.

Y. Iwamoto, M. Kohara, and M. Saito. On the consumption insurance effects of longterm care insurance in Japan: Evidence from micro-level household data. Journal of the Japanese and International Economies, 24:99-115, 2010.

A. J. Jacobs. Federations of municipalities: A practical alternative to local government consolidations in Japan? Governance: An International Journal of Policy, Administration, and Institutions, 17(2):247-274, 2004.

A. J. Jacobs. Japan's evolving nested municipal hierarchy: The race for local power in the 2000s. Urban Studies Research, 2011. Article ID 692764.

Japan Ministry of Health, Labour and Welfare (JMHLW). Annual Report on LTCI (Kaigo Hoken Jigyou Joukyou Houkoku, in Japanese). Tokyo, Various yearsa. 
Japan Ministry of Health, Labour and Welfare (JMHLW). Survey of Long-Term Care Management (Kaigo Jigyou Keiei Jittai Chousa in Japanese). Tokyo, Various yearsb.

Japan Ministry of Health, Labour and Welfare (JMHLW). Survey of Long-Term Care Benefit Expenditures (Kaigo Kyuufu-hi Jittai Chousa, in Japanese). Tokyo, Various yearsc.

Japan Ministry of Internal Affairs and Communications (JMIC). Annual Statistics on Municipal Tax Shi Cho Son Zei Kazei Joukyou Tou no Shirabe in Japanese). Tokyo, Various yearsa.

Japan Ministry of Internal Affairs and Communications (JMIC). Annual Statistics on Municipal Government Finance Shi Cho Son Betsu Kessan Joukyou Shirabe in Japanese). Tokyo, Various yearsb.

M. Johri, F. Beland, and H. Bergman. International experiments in integrated care for the elderly: a synthesis of the evidence. International Journal of Geriatric Psychiatry, 18:222-235, 2003.

R. Labelle, G. Stoddart, and T. Rice. A re-examination of the meaning and importance of supplier-induced demand. Journal of Health Economics, 13:347-368, 1994.

J. K. Malcomson. Health service gatekeepers. RAND Journal of Economics, 35:401-421, 2004.

T. G. McGuire. Physician agency. In A. J. Culyer and J. P. Newhouse, editors, Handbook of Health Economics 1. Elsevier, Amsterdam, 2000.

J. M. Mitchell and T. R. Sass. Physician ownership of ancillary services: Indirect demand inducement or quality assurance? Journal of Health Economics, 14:263-289, 1995.

J. Nakamura and S. Sugawara. A fallacy of a decreasing rate of parents-children coresidence: Increase of childless elders and their long-term care in Japan (in Japanese). Kikan Shakai Hosho Kenkyu, 51(3), 2016.

H. Noguchi and S. Shimizutani. Supplier density and at-home care use in Japan: Evidence from a micro-level survey on long-term care receivers. Japan and the World Economy, 21:365-372, 2009.

OECD. Long-term care for older people. Paris, 2005.

H. Rothgang. Social insurance for long-term care: An evaluation of the German model. Social Policy and Administration, 44(4):436-460, 2010.

A. Scott. Economics of general practice. In A. J. Culyer and J. P. Newhouse, editors, Handbook of Health Economics. Elsevier, Amsterdam, 2000.

R. SØrensen and J. Grytten. Competition and supplier-induced demand in a health care system with fixed fees. Health Economics, 8:497-508, 1999. 
S. Sugawara. Firm-driven management for longevity risk: Analysis of lump-sum forward payments in Japanese nursing homes. SSRN Working Paper 2674426, 2015.

S. Sugawara and J. Nakamura. Can formal elderly care stimulate female labor supply? the Japanese experience. Journal of the Japanese and International Economies, 34: 98-115, 2014.

H. Sumii and Y. Sawada, editors. International dictionary of long-term care insurance terms: Grobalization of long-term care insurance (Kokusai kaigo hoken yougo jiten: Kaigo hoken no kokusai ka, in Japanese). Daigaku Kyouiku Shuppan, 2012.

W. Suzuki. Are there advantages for nonprofit home service providers? (in Japanese). Kikan Shakai Hosho Kenkyu, 38(1):74-88, 2002.

N. Tamiya, H. Noguchi, A. Nishi, M. R. Reich, N. Ikegami, H. Hashimoto, K. Shibuya, I. Kawauchi, and J. C. Campbell. Population ageing and wellbeing: lessons from japan's long-term care insurance policy. Lancet, 378:1183-1192, 2011.

The Council of Local Authorities for International Relations (CLAIR) and The National Graduate Institute for Policy Studies (GRIPS). Statistics on Local Governance (Japanese and English). Tokyo, 2010.

T. Tsutsui and N. Muramatsu. Japan's universal long-term care system reform of 2005: Containing costs and realizing a vision. Journal of the American Geriatrics Society, 55(9):1458-1463, 2007.

C. H. van Houtven and E. C. Norton. Informal care and health care use of older adults. Journal of Health Economics, 23:1159-1180, 2004.

Y. Yamauchi. Home visit care expenses and provider density (Homon kaigo hi to jigyosha mitsudo in Japanese). Iryo to Shakai, 14(2):103-118, 2004.

V. Yong and Y. Saito. Trends in healthy life expectancy in Japan: 1986-2004. Demographic Research, 20:467-494, 2009.

M. Yuda. The effect of provider density on long-term care, (Kaigo jigyosha mitsudo ga kaigo service ni ataeru eikyo, in Japanese). Kikan Shakai Hosho kenkyu, 40(4): 378-386, 2005.

\section{Appendix}

\section{Remuneration points for care management}

This appendix summarizes remuneration points of home-based care management. The LTCI was revised every three years, and there were four revisions between 2000 and 2013. In the first season of the LTCI from April 2000 to March 2003, care management was assigned different remuneration points according to the LTCI classification level of the elderly, which is determined based on care requirements. There were six classification 
levels in the first season, $\mathrm{AR}$ and $\mathrm{CR} 1$ to 5 , where the latter levels required more intensive care. The rewards for care plans were 650 points for AR; 720 points for CR 1 and 2; and 840 points for CR 3, 4, and 5 .

In the second season between April 2003 and March 2006, price discrimination through the classification level was eliminated and all care plans were rewarded 850 points. Instead, a bonus and a penalty were introduced. As a bonus, additional 100 points were assigned for care plans that combined four or more services. As a penalty, $30 \%$ of rewards were canceled in case of violation of rules.

The third season between April 2006 and March 2009 drastically changed as a result of the major amendment of the LTCI in 2006. Price discrimination through the classification level was revived. The rewards for care plans were 1000 points for CR 1 and 2; 1300 points for CR 3, 4, and 5; and 850 points for transitive CR. AR was transferred to the preventive care sector, but home-based care management offices could see preventive care consumers upon request from the community comprehensive care center. The care plan for preventive care was rewarded 400 points.

There were bonuses for entire offices as well as each care plan. Offices who satisfied several requirements that proved hardship and quality of care management received additional 200 points for all care plans. Care plans for consumers in specific regions, like isolated islands and heavy snow areas, received extra 15\% reward. Additional 250 points were assigned for care plans for new consumers or consumers who experienced an instantaneous rise of classification levels in two or more levels. Further, additional 600 points were allocated for care plans for those who returned home from hospitals or institutional care sectors after staying for 30 days or more. The bonus for combination of four or more services was eliminated.

There were three penalties. First, the penalty for violation of rules continued from the previous season, and became more severe. After one month with $30 \%$ reward reduction, offices faced an increased penalty of $50 \%$ reward reduction in subsequent months if the situation was not fixed. The second was the penalty of volume. The reward was decreased to $60 \%$ for each care plan if home-based care management offices created between 40 and 59 care plans per care manager on an average. The reward was further decreased to $40 \%$ if offices created 60 or more care plans. A consumer of the preventive care management is counted as 0.5 people and a consumer was allowed to create up to eight preventive care plans. The third was the penalty for concentration of providers. 200 points were decreased for all care plans if a care management office employed a specific provider in more than $90 \%$ of their care plans in each half year, without a comprehensive reason. More details of this penalty are described in Section 2.

In the third season between April 2009 and March 2012, many bonuses were revised and added. For revised bonuses, the bonuses for offices to prove hardship and quality were separated to two levels, 500 points for the first-level offices and 300 points for the second-level offices. The bonus for new consumers was increased to 300 points. The bonuses of care plans after hospitalization was also separated to two levels, 400 points for less than 30 days of stay and 600 points for 30 days or more.

Bonuses for operation in mesomountaineous (Chuusankan) areas were introduced as additional 10 points for offices located in such areas and additional 5 points for care plans 
for consumers living in those areas. New bonuses were introduced for difficult care plans. Plans for dementia patients and for single dwelling elderly persons received additional 150 points. Collaboration with the medical sector and multifunctional long-term care in a small group home were rewarded with additional 150 and 300 points, respectively. On the other hand, the volume penalty became more severe. The reward was decreased to $50 \%$ and $30 \%$ for offices with 40 to 59 care plans per care manager or 60 or more care plans, respectively.

In the fourth season between April 2012 and March 2015, the bonus for collaboration with a medical sector is separated for the first and second classes that are rewarded 200 and 100 points, respectively. To obtain the first class, a care manager needs to make a physical visit to the hospital. The bonus of care plans after hospitalization has been made uniform; however, this bonus can now be assigned for at most three times for each consumer. A new bonus of 200 points has been introduced for holding an emergency conference of service providers. Additionally, collaboration with a new service called multiple-service providers has been assigned a bonus of 300 points. The penalty for concentration of providers has become more severe and a penalty of $100 \%$ is imposed from the second month.

\section{Tables}




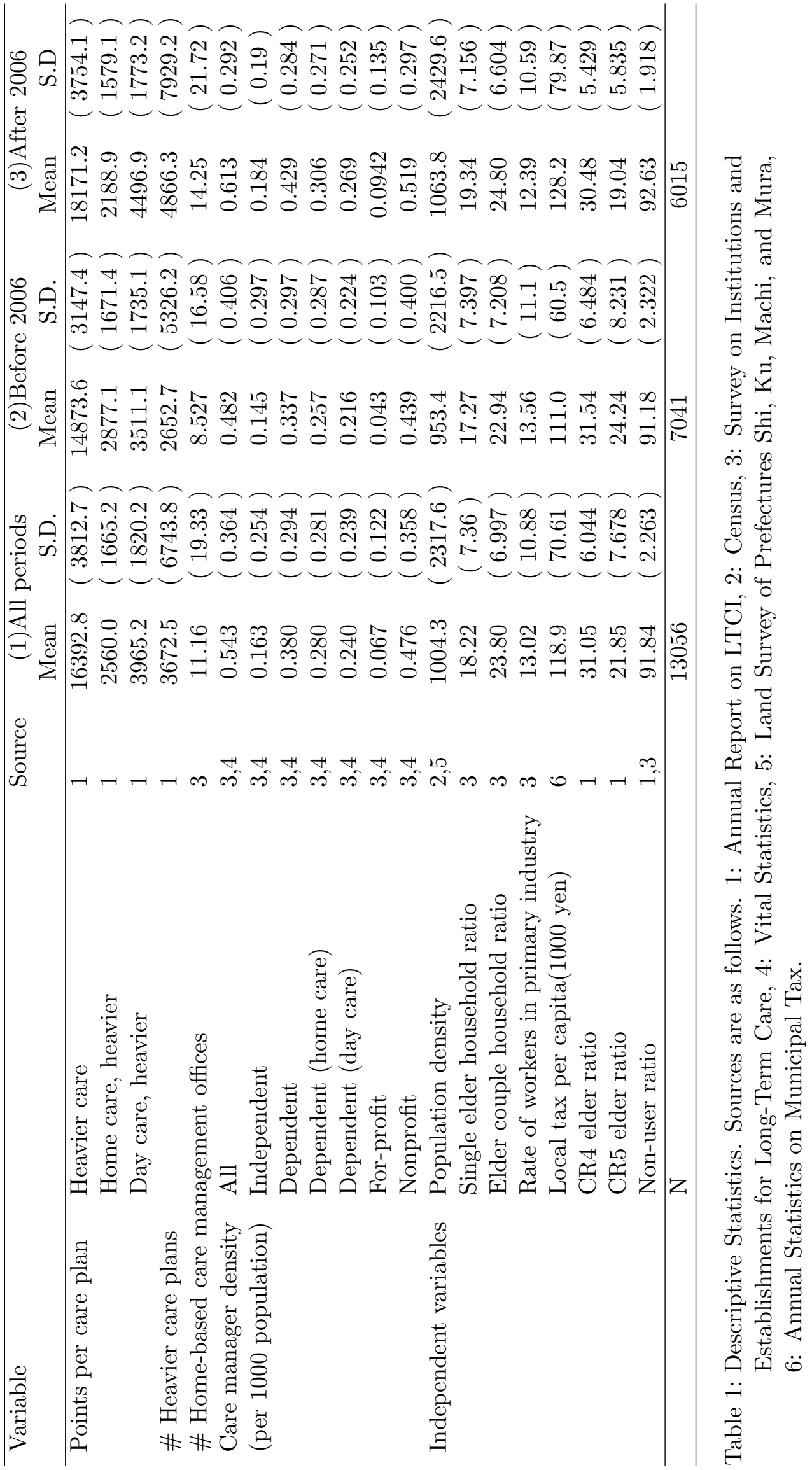




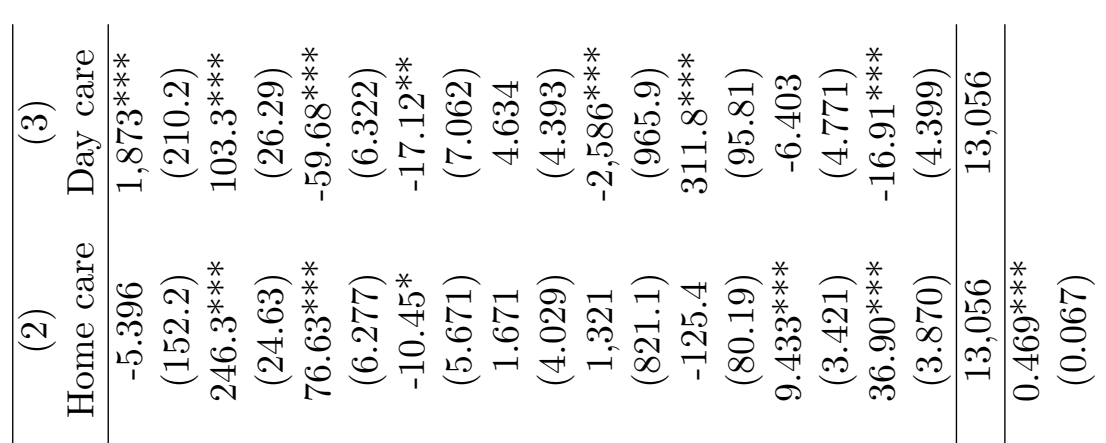

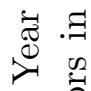

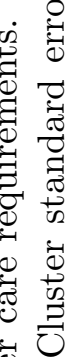

$\overrightarrow{8}$
$\dot{0}$
$\dot{1}$

范

范范

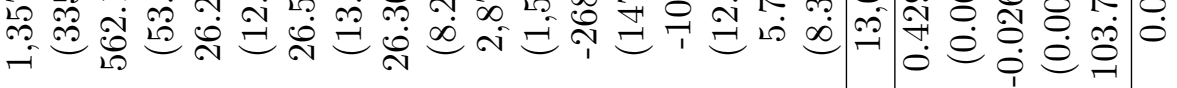

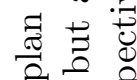

ए

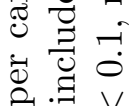

in 02



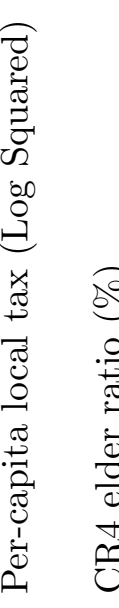

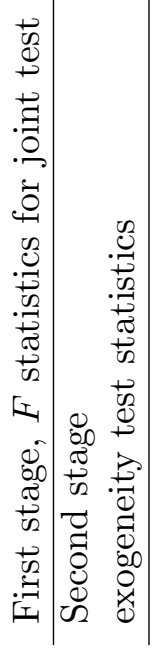

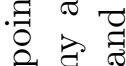

윰ำ

莺

曾

ซี $\frac{\pi}{\overline{0}} \sqrt{0}$

ग्ठ․

:

:

:

告曽*

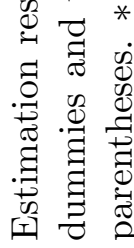

$\ddot{i}$
$\stackrel{0}{0}$
$\stackrel{\pi}{\pi}$ 


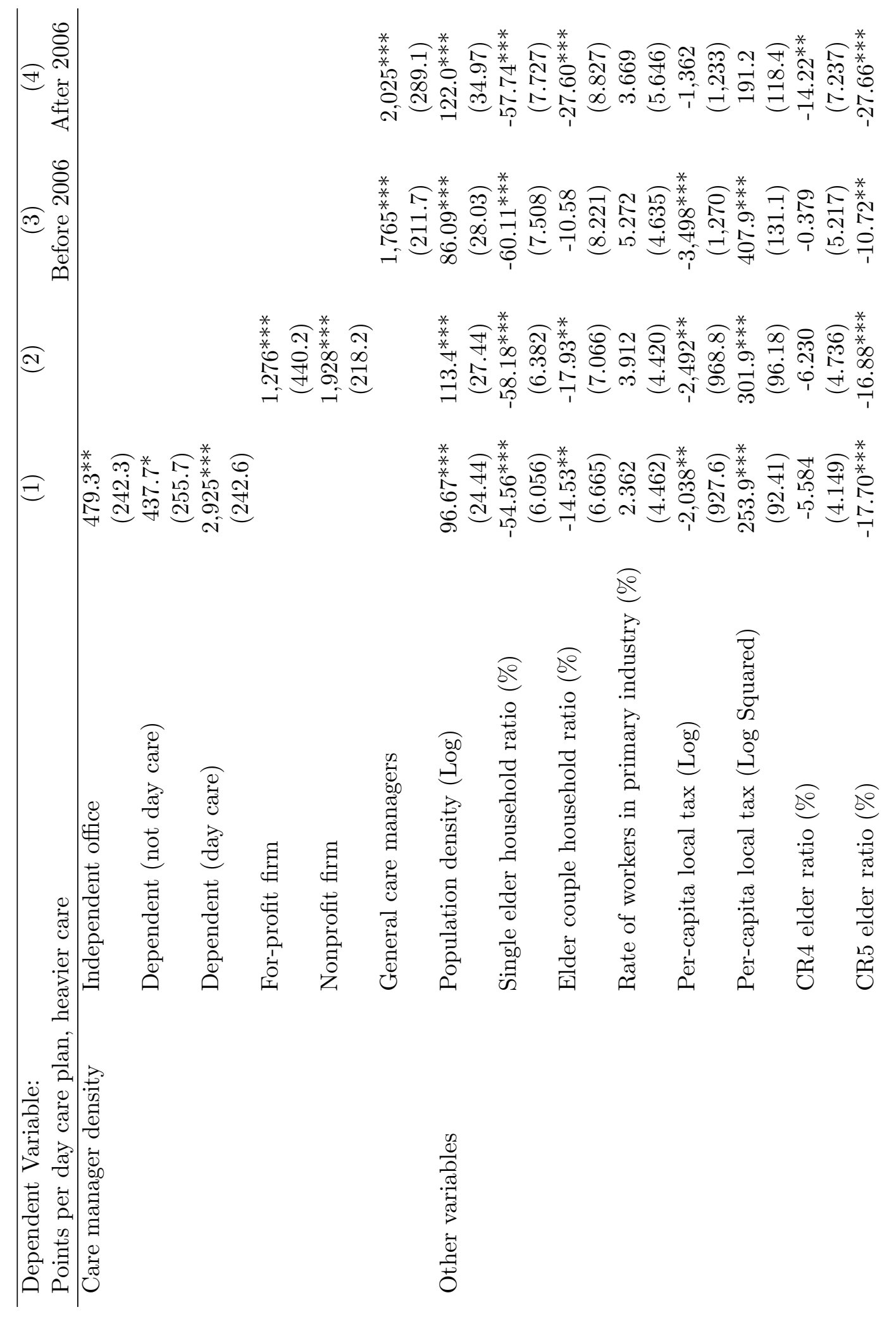




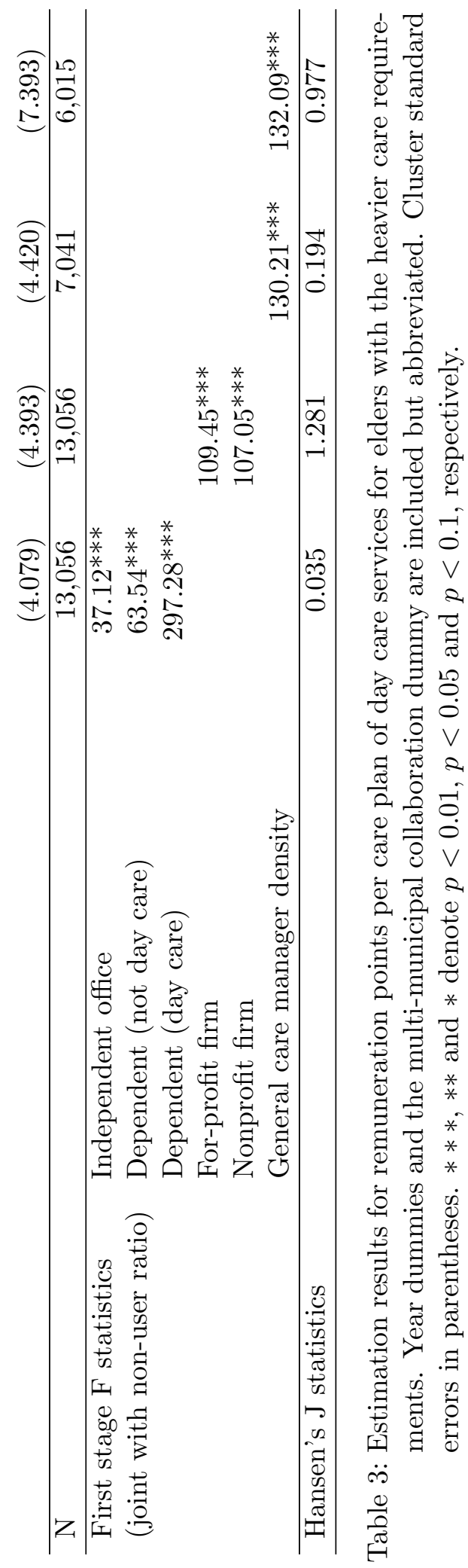




\begin{tabular}{llcc}
\hline & & $\begin{array}{c}\text { Difference } \\
(1)\end{array}$ & $\begin{array}{c}\chi^{2} \text { statistics } \\
(2)\end{array}$ \\
\hline Analysis 1 & All periods & 2463.1 & $98.3^{* * *}$ \\
Analysis 2 & 2002 & 2036.11 & $88.58^{* * *}$ \\
& 2003 & 3072.6 & $118.54^{* * *}$ \\
& 2004 & 3039.1 & $80.18^{* * *}$ \\
& 2005 & 2810.31 & $85.47^{* * *}$ \\
& 2006 & 262 & 0.26 \\
& 2007 & 2281.4 & $46.31^{* * *}$ \\
& 2008 & 1871.1 & $44.96^{* * *}$ \\
& 2009 & 1754.4 & $38.61^{* * *}$ \\
\hline
\end{tabular}

Table 4: Estimation results for robustness check. Column (1) show differences of coefficient estimates for dependent and non-dependent care manager densities. Column (2) shows $\chi^{2}$ test statistics for the hypothesis that the difference is zero. $* * *, * *$ and $*$ denote $p<0.01, p<0.05$ and $p<0.1$, respectively. 